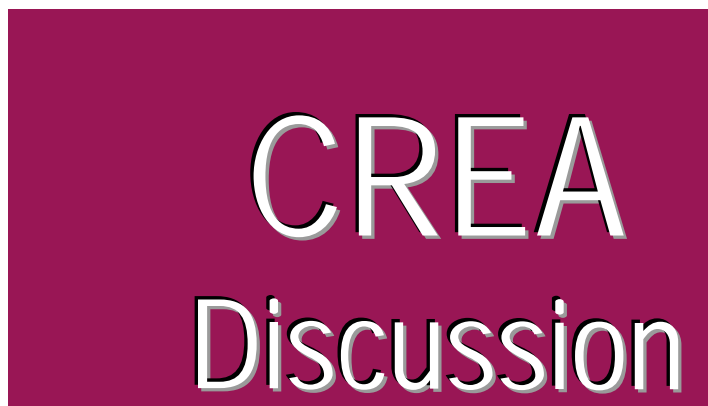
Paper

\title{
2013-26
}

Center for Research in Economic Analysis

University of Luxembourg

\section{A Generalized Steady-State Growth Theorem}

available online : http://wwwfr.uni.lu/recherche/fdef/crea/publications2/discussion_papers

Andreas Irmen, CREA, Université du Luxembourg

November, 2013

For editorial correspondence, please contact: crea@uni.lu

University of Luxembourg Faculty of Law, Economics and Finance 162A, avenue de la Faïencerie

L-1511 Luxembourg 


\title{
A GENERALIZED
}

\section{STEADY-STATE GROWTH THEOREM}

\author{
Andreas Irmen* \\ University of Luxembourg and CESifo, Munich
}

\begin{abstract}
Uzawa's steady-state growth theorem (Uzawa (1961)) is generalized to a neoclassical economy that uses current output, e. g., to create technical progress or to manufacture intermediates. The difference between aggregate final-good production and these resources is referred to as net output. The new generalized steady-state growth theorem holds since net output exhibits constant returns to scale in capital and labor. This insight provides an understanding for why technical change is labor-augmenting in steady state even if capital-augmenting technical change is feasible. By example, this point is made for three recent growth models that allow for endogenous capital- and labor-augmenting technical change, namely, Irmen (2013), Acemoglu (2003), and Acemoglu (2009), Chapter 15. The reduced form of these models is shown to be consistent with the generalized steady-state growth theorem.
\end{abstract}

Keywords: Steady-State Growth, Capital Accumulation, Uzawa's Theorem, Endogenous Direction of Technical Change.

JEL-Classification: E10, O10, O40.

This Version: November 8, 2013.

\footnotetext{
*University of Luxembourg, Faculty of Law, Economics and Finance, 162a, avenue de la Faïencerie, L-1511 Luxembourg, Luxembourg, airmen@uni.lu.

I would like to thank Anastasia Litina, Amer Tabakovic, Gautam Tripathi, Bertrand Wigniolle, and Benteng Zou for helpful comments.
} 


\section{Introduction}

Why is technical change almost always labor-augmenting in Dynamic Macroeconomics? For the broad class of neoclassical growth models, Uzawa's steady-state growth theorem suggests the answer (Uzawa (1961)). ${ }^{1}$ Roughly speaking, this theorem says that only labor-augmenting technical change is consistent with the steady-state growth path of a neoclassical economy. The theorem exploits two fundamental components of neoclassical growth theory, namely, the aggregate production function with constant returns to scale in capital and labor and the role of capital accumulation. Hence, the desire for a dynamic macro-economy to exhibit a steady state and the analytical structure of neoclassical growth models suggest right from the start the assumption of an aggregate production function with labor-augmenting technical change.

Uzawa derived his insight with a view to a neoclassical economy where technical progress is exogenous and costless. The generalized steady-state growth theorem established in the present paper extends this insight to an economy where current output is used to generate technical progress or to manufacture intermediates. I refer to the latter resources as aggregate investment. Typically, this additional element matters in an economy where technical progress is endogenous and costly. Moreover, it gives rise to the notion of net output defined as the difference between aggregate final-good production and aggregate investment.

Hence, the neoclassical economy under scrutiny here comprises an aggregate production function, an aggregate investment function, a resource constraint, and an equation of motion describing the accumulation of capital. For such an economy, the generalized steady-state growth theorem characterizes steady-state paths starting in finite time. The first part of the theorem establishes that net output, aggregate output, aggregate investment, capital, and aggregate consumption grow at the same rate. Its second part shows that technical change is purely labor-augmenting in the net output function. Moreover, the growth rate of laboraugmenting technical change is shown to coincide with the growth rate of all

\footnotetext{
${ }^{1}$ A steady state is defined as a path along which all variables grow at constant exponential rates. These rates may be positive, zero, or negative. Schlicht (2006) provides an elegant and intuitive proof of Uzawa's theorem which was successively adopted by Jones and Scrimgeour (2008) and Acemoglu (2009). The proof of the new generalized steady-state growth theorem developed below builds on and extends Schlicht's proof strategy.
} 
per-worker variables. While the proof of the first part follows directly from the analytical structure of the model, its second part is shown to rely on the assumption that both the aggregate production function and the aggregate investment function exhibit constant returns to scale in capital and labor.

From the perspective of these results I take a new look at the question about why steady-state technical change is labor-augmenting in endogenous growth models that allow for capital- and labor-augmenting technical change and involve capital accumulation. By way of three examples, I argue that in steady state the reduced form of these models either involves a net output function that has constant returns in capital and labor as required by the generalized steady-state growth theorem or is consistent with Uzawa's original formulation. Therefore, in steady state capital-augmenting technical change vanishes and labor-augmenting technical change determines the growth rate of the economy. ${ }^{2}$

This point is made for the one-sector model of Irmen (2013) as well as for the multi-sector model of Acemoglu (2003) and its extension (Acemoglu (2009), Chapter 15). ${ }^{3}$ To the best of my knowledge, these studies are the only growth models in the literature allowing for endogenous capital- and labor-augmenting technical change and capital accumulation. ${ }^{4}$

For the question at hand, it matters that these models differ in the way technical change is generated. In Irmen (2013) this requires the input of current final-good production. Therefore, the generalized steady-state growth theorem can be applied. In Acemoglu's two variants technical change is the result of research conducted by labor. For Acemoglu (2003), this is shown to lead to an application of Uzawa's original theorem. As current final output is used up as an input in the production of intermediates in Acemoglu (2009), aggregate investment is strictly positive. Therefore, the generalized steady-state growth theorem is shown to apply.

The remainder of this paper is organized as follows. Section 2 has the statement and the proof of the generalized steady-state growth theorem. Section 2.1 gives

\footnotetext{
${ }^{2}$ Klump, McAdam, and Willman (2007) study the US economy and confirm this pattern of technical change empirically for the period 1953 to 1998.

${ }^{3}$ The reduced form of the three-sector model of endogenous capital- and labor-augmenting technical change devised in Irmen (2011) shares the relevant properties with the one-sector model of Irmen (2013). Hence, all findings derived in Section 3.1 also apply to this three-sector model.

${ }^{4}$ Jones (2005) develops an alternative argument for why technical change is labor-augmenting.
} 
the precise setup of the neoclassical economy under scrutiny. The generalized steady-state growth theorem appears as Theorem 1 in Section 2.2. Section 2.3 discusses important assumptions and features of it. They include the role of differing technologies affecting aggregate production and aggregate investment, the link to Uzawa's original result, the importance of capital accumulation and of constant returns. Finally, I turn to the special, yet important case of factor-augmenting technologies. Section 3 establishes the link between the generalized steady-state growth theorem and the steady-state properties of the above mentioned endogenous growth models. Their order of appearance reflects an increasing degree of complexity. Section 4 concludes this paper. If not indicated otherwise proofs are relegated to the Appendix.

\section{Statement and Proof of the Theorem}

\subsection{The Model}

Consider a closed economy, and, without loss of generality, let time be continuous, i. e., $t \in(-\infty,+\infty)$. The production sector consists of two elements. First, there is an aggregate production function of the final good

$$
Y(t)=\tilde{F}\left[K(t), L(t), \mathbf{A}_{F}(t)\right],
$$

where $\tilde{F}: \mathbb{R}_{+}^{2} \times \mathfrak{A}_{F} \rightarrow \mathbb{R}_{+}, Y(t)$ is aggregate output of the final good, $K(t)>0$ is the capital stock, $L(t)>0$ is the labor endowment, and $\mathbf{A}_{F}(t) \in \mathfrak{A}_{F}$ represents the components of technological knowledge available at $t$ that affect the production of the final good. Here, $\mathfrak{A}_{F}$ is an arbitrary set. ${ }^{5}$

Second, there is an aggregate investment function. It states the amount of period$t$ final-good output that is used up in the same period as an input somewhere in the economy. For instance, the economy may invest contemporaneous final output to generate technical progress in its research sector or, alternatively, use it as an input in an intermediate-good industry of the production sector. In any case, the defining property of these resources is that they are neither available for

\footnotetext{
${ }^{5}$ In general, the specification of $\mathfrak{A}_{\mathfrak{F}}$ (and $\mathfrak{A}_{\mathfrak{J}}$ introduced below) will depend on how technological knowledge and its components are represented.
} 
consumption nor for the accumulation of capital. I refer to them as "aggregate investment" denoted by $I(t)$. Let

$$
I(t)=\tilde{I}\left[K(t), L(t), \mathbf{A}_{I}(t)\right],
$$

where $\tilde{I}: \mathbb{R}_{+}^{2} \times \mathfrak{A}_{I} \rightarrow \mathbb{R}_{+}$is the aggregate investment function, and $\mathbf{A}_{I}(t) \in \mathfrak{A}_{I}$ represents components of technological knowledge available at $t$ that affect the amount of invested final output given capital and labor. Again, $\mathfrak{A}_{I}$ is an arbitrary set.

In most applications the functions $\tilde{F}$ and $\tilde{I}$ will correspond to reduced-form production and investment functions of the economy under scrutiny. As such, they will reflect the optimal behavior of economic actors and the market-clearing conditions. This justifies the assumption that these functions depend both on the capital and the labor endowment of the economy. ${ }^{6}$ However, there is little reason why the technology applied in the production of the final good should coincide with the technology used in the economy's research or intermediate-good sector. This is why $\mathbf{A}_{F}(t)$ is allowed to differ from $\mathbf{A}_{I}(t)$.

Suppose that both $\tilde{F}$ and $\tilde{I}$ are increasing in $K(t)$ and $L(t)$ and exhibit constant returns to scale in these arguments. Then, $V(t)=Y(t)-I(t)$ is net output, i. e.,

$$
\begin{aligned}
V(t) & =\tilde{F}\left[K(t), L(t), \mathbf{A}_{F}(t)\right]-\tilde{I}\left[K(t), L(t), \mathbf{A}_{I}(t)\right] \\
& \equiv \tilde{V}\left[K(t), L(t), \mathbf{A}_{F}(t), \mathbf{A}_{I}(t)\right]
\end{aligned}
$$

where $\tilde{V}: \mathbb{R}_{+}^{2} \times \mathfrak{A}_{F} \times \mathfrak{A}_{I} \rightarrow \mathbb{R}_{+}$exhibits constant returns to scale in $K(t)$ and $L(t)$, too. Hence, net output is defined as the amount of the final good that is available for consumption and capital accumulation. Henceforth, I refer to $\tilde{V}$ as the net output function. Capital and aggregate consumption, $C(t)$, are measured in units of the final good. Then, at all $t$ capital accumulates according to

$$
\dot{K}(t)=V(t)-C(t)-\delta_{K} K(t), \quad \delta_{K} \in \mathbb{R}_{+},
$$

where $\delta_{K}$ is the instantaneous depreciation rate of capital. Finally, the evolution of the labor endowment is given by

$$
L(t)=L(0) e^{g_{L} t}, \quad L(0)>0, \quad g_{L} \in \mathbb{R},
$$

${ }^{6}$ This assumption will be relaxed in Section 2.3 .4 below. 
i. e., the instantaneous growth rate of the labor force is time-invariant and may be positive, zero, or negative.

In what follows, I denote by $g_{x}(t) \in \mathbb{R}$ the instantaneous growth rate of a variable $x(t)$ at $t$. By definition, a steady state has $g_{x}(t)=g_{x}$ for all variables featured in the model.

\subsection{The Generalized Steady-State Growth Theorem}

Theorem 1 Consider an economy described by equations (2.3), (2.4), and (2.5). Suppose there exists a steady-state path starting at some date $\tau<\infty$ such that $Y(t)>V(t)>$ $C(t)>0$ for all $t \geq \tau$. Then, the following holds:

I. $g_{V}=g_{Y}=g_{I}=g_{K}=g_{C}$.

II. For any $t \geq \tau$, net output has a representation as

$$
V(t)=V[K(t), A(t) L(t)],
$$

where $A(t)=e^{\left(g_{V}-g_{L}\right)(t-\tau)} \in \mathbb{R}_{++}$, and

$$
g=g_{V}-g_{L}
$$

is the growth rate of per-worker variables.

The main message of the generalized steady-state growth theorem is that steadystate technical change is labor-augmenting in the net output function. Moreover, the growth rate at which the technology evolves determines the growth rate of all per-worker variables. This insight comes in two steps.

Part I shows that the steady-state growth rates of net output, aggregate finalgood output, aggregate investment, capital, and aggregate consumption are the same. This follows since in steady state a strictly positive difference between two strictly positive variables satisfies that the growth rates of the minuend and the subtrahend coincide. Both, the definition of net output and of steady-state capital accumulation give rise to such differences (see equations (5.1) and (5.2) in the Proof of Theorem 1). In the present context, this property has two implications. 
First, net output requires $g_{V}=g_{Y}=g_{I}$ since $Y(t)>I(t)>0$. Second, steadystate capital accumulation requires $g_{V}=g_{K}=g_{C}$ since $V(t)>C(t)>0$.

Part II exploits constant returns to capital and labor in the net output function in conjunction with $g_{V}=g_{K}$, the requirement of steady-state capital accumulation. Together, these properties imply steady-state labor-augmenting technical change with a growth rate equal to $g_{V}-g_{L}$. Labor-augmenting technical change at this rate assures that the first two arguments in $\tilde{V}$ of (2.3) - with respect to which $\tilde{V}$ has constant returns to scale - grow at the same rate. Accordingly, the steady state has $g_{V}=g_{K}=g+g_{L}$ and, in light of Part I, $g$ is the growth rate of all per-worker variables.

Whether per-worker variables grow or shrink hinges on how $g_{V}=g_{K}$ relates to the exogenous growth rate of the labor force, $g_{L}$. If $g_{V}=g_{K}=g_{L}$ then $g=0$, i. e., there is no technical change and per-worker variables remain constant over time. If $g_{V}=g_{K}>g_{L}$ then capital grows faster than labor and strictly positive labor-augmenting technical change makes up for the difference. Moreover, perworker variables grow at the rate of technical change. Finally, if $g_{V}=g_{K}<g_{L}$ then capital grows slower than labor and labor-augmenting technical change is negative to close the gap. In this case, per-worker variables shrink at the rate of technical decline.

\subsection{Discussion}

\subsubsection{Technical Change in $Y(t)$ and $I(t)$}

In the economy under scrutiny here the technology may affect aggregate production and aggregate investment in different ways, i. e., $\mathbf{A}_{F}(t) \neq \mathbf{A}_{I}(t)$. However, in light of (2.3) the generalized steady-state growth theorem implies the existence of two linear homogeneous functions, $F: \mathbb{R}_{+}^{2} \rightarrow \mathbb{R}_{+}$and $I: \mathbb{R}_{+}^{2} \rightarrow \mathbb{R}_{+}$, such that

$$
Y(t)=F[K(t), A(t) L(t)] \quad \text { and } \quad I(t)=I[K(t), A(t) L(t)]
$$

for all $t \geq \tau$ where $A(t)=e^{\left(g_{V}-g_{L}\right) t} \in \mathbb{R}_{++}$. Hence, even though $\mathbf{A}_{F}(\tau) \neq \mathbf{A}_{I}(\tau)$ may hold, steady-state technical change must be labor-augmenting and evolve at the same pace in both the aggregate production and the aggregate investment function. 


\subsubsection{What if $I(t)=0$ ?}

The generalized steady-state growth theorem postulates an aggregate investment function that takes on strictly positive values for all $t \geq \tau$. As shown in Section 3 below, this extension is important to understand the key structural properties of models with endogenous capital- and labor-augmenting technical change. Absent an aggregate investment function, i. e., if $I(t)=0$ for all $t \geq \tau$, the distinction between gross and net output vanishes. In this case, Theorem 1 and Uzawa's original theorem coincide.

\subsubsection{What if $V(t)=C(t)>0$ ?}

Capital accumulation is a central ingredient to the generalized steady-state growth theorem. Indeed, the assumption that $V(t)>C(t)>0$ for all $t \geq \tau$ assures that some final output is always used to accumulate capital. If instead $V(t)=C(t)>$ 0 holds, then no final output is allocated to the accumulation of capital. The following corollary highlights the necessary changes to Theorem 1.

Corollary 1 Reconsider the economy described by equations (2.3), (2.4), and (2.5). Suppose there exists a steady-state path starting at some date $\tau<\infty$ such that $Y(t)\rangle$ $V(t)=C(t)>0$ for all $t \geq \tau$. Then, the following holds:

I. $g_{V}=g_{Y}=g_{I}=g_{C}$ and $g_{K}=-\delta_{K}$.

II. For any $t \geq \tau$, net output has a representation as

$$
V(t)=V[B(t) K(t), A(t) L(t)],
$$

where $B(t)=e^{\left(g_{V}+\delta_{K}\right)(t-\tau)} \in \mathbb{R}_{++}$and $A(t)=e^{\left(g_{V}-g_{L}\right)(t-\tau)} \in \mathbb{R}_{++}$.

Capital per worker grows at rate $-\left(\delta_{K}+g_{L}\right)$. All remaining per-worker variables grow at rate $g=g_{V}-g_{L}$. 
Corollary 1 states that steady-state capital-augmenting technical change does not necessarily disappear in a world without capital accumulation. The intuition for this comes in two steps. As to Part I, the new feature is that (2.4) and $V(t)=$ $C(t)$ now imply $g_{K}=-\delta_{K}$ and $g_{V}=g_{C}$. Hence, the evolution of net output is decoupled from the evolution of capital whereas steady-state growth of net output still requires $g_{V}=g_{Y}=g_{I}$ since $Y(t)>I(t)>0$.

Part II shows that this decoupling requires capital-augmenting technical change at rate $g_{V}+\delta_{K}$ to have "efficient capital" and "efficient labor" grow at the same rate in the net output function $\tilde{V}$ of (2.3). Capital-augmenting technical change disappears only if $g_{V}=-\delta_{K}$, the case in which net output and capital grow at the same rate.

Clearly, the growth rate of capital per worker is $-\left(\delta_{K}+g_{L}\right)$. As $g_{V}=g+g_{L}$, the growth rate of all other per-worker variables is given by $g$, the growth rate of labor-augmenting technical change.

\subsubsection{Constant Returns to Capital and Labor in $\tilde{F}$ and $\tilde{I}$}

Constant returns to capital and labor in the net output function (2.3) is key to the generalized steady-state growth theorem. However, for this to hold it is not necessary that both $\tilde{F}$ and $\tilde{I}$ share this property. In fact, Theorem 1 does not change if we allow for the aggregate production and/or the aggregate investment function to be linear in either capital or labor. ${ }^{7}$ Doing so gives rise to the following six

\footnotetext{
${ }^{7}$ Observe that unlike Theorem 1, the character of Uzawa's original theorem drastically changes if the aggregate production function becomes either linear in labor or in capital. In the former case, $Y(t)=L(t) \tilde{\tilde{F}}\left(\mathbf{A}_{F}(t)\right)$ and, void of capital and its accumulation, steady-state technical change has to be labor-augmenting. In the latter case, $Y(t)=K(t) \tilde{\tilde{F}}\left(\mathbf{A}_{F}(t)\right)$, i. e., aggregate production is of the $A K$-type. Here, labor is not explicitly accounted for and the mere notion of labor-augmenting technical change becomes pointless.
} 
variants:

$$
V(t)=\left\{\begin{array}{cc}
L(t) \tilde{\tilde{F}}\left(\mathbf{A}_{F}(t)\right)-\tilde{I}\left[K(t), L(t), \mathbf{A}_{I}(t)\right], & \text { or } \\
\tilde{F}\left[K(t), L(t), \mathbf{A}_{F}(t)\right]-L(t) \tilde{\tilde{I}}\left(\mathbf{A}_{I}(t)\right), & \text { or } \\
K(t) \tilde{\tilde{F}}\left(\mathbf{A}_{F}(t)\right)-\tilde{I}\left[K(t), L(t), \mathbf{A}_{I}(t)\right], & \text { or } \\
\tilde{F}\left[K(t), L(t), \mathbf{A}_{F}(t)\right]-K(t) \tilde{\tilde{I}}\left(\mathbf{A}_{I}(t)\right), & \text { or } \\
L(t) \tilde{\tilde{F}}\left(\mathbf{A}_{F}(t)\right)-K(t) \tilde{\tilde{I}}\left(\mathbf{A}_{I}(t)\right), & \text { or } \\
K(t) \tilde{\tilde{F}}\left(\mathbf{A}_{F}(t)\right)-L(t) \tilde{\tilde{I}}\left(\mathbf{A}_{I}(t)\right), &
\end{array}\right.
$$

where $\tilde{\tilde{F}}: \mathfrak{A}_{F} \rightarrow \mathbb{R}_{++}$and $\tilde{\tilde{I}}: \mathfrak{A}_{I} \rightarrow \mathbb{R}_{++}$. Intuitively, for all these specifications Part I of Theorem 1 goes through since its proof relies only on the analytical structure of the underlying model and not on functional forms. Moreover, Part II of Theorem 1 remains valid since it relies on constant returns of the net output function, a property that all specifications of (2.6) preserve.

\subsubsection{Factor-Augmenting Technical Change}

Technical change is factor-augmenting in the aggregate production function and the aggregate investment function if and only if these aggregates can be put into the form

$$
Y(t)=F\left[B_{F}(t) K(t), A_{F}(t) L(t)\right] \quad \text { and } \quad I(t)=I\left[B_{I}(t) K(t), A_{I}(t) L(t)\right] ;
$$

here $B_{j}(t) \in \mathbb{R}_{++}$and $A_{j}(t) \in \mathbb{R}_{++}, j=F, I$, represent the capital- and the laboraugmenting technology in the respective aggregate. Compared to the general specification of technical change that appears in $\tilde{F}$ and $\tilde{I}$ of (2.1) and (2.2), the stipulation that technical change has to be factor-augmenting is restrictive. In a sense, it assumes the "form of technical change" that results as an implication in Theorem 1. However, imposing factor-augmenting technical change also leads to additional insights as it allows for the identification of circumstances where technical change involving growth rates $g_{B_{F}} \neq 0$ and $g_{B_{I}} \neq 0$ is consistent with an overall representation of technical change as labor-augmenting. The following corollary sharpens this statement further. 
Corollary 2 Consider an economy comprising net output

$$
V(t)=F\left[B_{F}(t) K(t), A_{F}(t) L(t)\right]-I\left[B_{I}(t) K(t), A_{I}(t) L(t)\right]
$$

and equations (2.4) and (2.5). Suppose there exists a steady-state path starting at some date $\tau<\infty$ such that $Y(t)>V(t)>C(t)>0$ for all $t \geq \tau$. Then, the following holds:

1. If $g_{B_{F}}=g_{B_{I}}=0$ then $g=g_{A_{F}}=g_{A_{I}}$.

2. If $g_{B_{F}}=0$ and $g_{B_{I}} \neq 0$ then net output has the form

$$
V(t)=F\left[B_{F}(\tau) K(t), A_{F}(t) L(t)\right]-\beta_{I} K(t)^{\alpha_{I}}\left(e^{g(t-\tau)} L(t)\right)^{1-\alpha_{I}},
$$

where $0<\alpha_{I}<1, \beta_{I}=c_{I} B_{I}(\tau)^{\alpha_{I}} A_{I}(\tau)^{1-\alpha_{I}}>0$, and

$$
g=\frac{\alpha_{I} g_{B_{I}}}{1-\alpha_{I}}+g_{A_{I}}=g_{A_{F}} \text {. }
$$

3. If $g_{B_{F}} \neq 0$ and $g_{B_{I}}=0$ then net output has the form

$$
V(t)=\beta_{F} K(t)^{\alpha_{F}}\left(e^{g(t-\tau)} L(t)\right)^{1-\alpha_{F}}-I\left[B_{I}(\tau) K(t), A_{I}(t) L(t)\right],
$$

where $0<\alpha_{F}<1, \beta_{F}=c_{F} B_{F}(\tau)^{\alpha_{F}} A_{F}(\tau)^{1-\alpha_{F}}>0$, and

$$
g=\frac{\alpha_{F} g_{B_{F}}}{1-\alpha_{F}}+g_{A_{F}}=g_{A_{I}} \text {. }
$$

4. If $g_{B_{F}} \neq 0$ and $g_{B_{I}} \neq 0$ then net output has the form

$$
V(t)=\beta_{F} K(t)^{\alpha_{F}}\left(e^{g(t-\tau)} L(t)\right)^{1-\alpha_{F}}-\beta_{I} K(t)^{\alpha_{I}}\left(e^{g(t-\tau)} L(t)\right)^{1-\alpha_{I}},
$$

and

$$
g=\frac{\alpha_{I} g_{B_{I}}}{1-\alpha_{I}}+g_{A_{I}}=\frac{\alpha_{F} g_{B_{F}}}{1-\alpha_{F}}+g_{A_{F}}
$$

The upshot of Corollary 2 is that steady-state technical change may involve $g_{B_{F}} \neq$ 0 and/or $g_{B_{I}} \neq 0$. However, this is only permissible if the respective aggregate is Cobb-Douglas and the growth rates $g_{B_{F}}, g_{A_{F}}, g_{B_{I}}$, and $g_{A_{I}}$ are aligned such that aggregate production and aggregate investment grow at the same rate.

Claim 1 is a benchmark and immediate from Theorem 1. If $g_{B_{F}}=g_{B_{I}}=0$ then technical change is labor-augmenting in $F$ and $I$ and evolves at the same pace, $g$, which is also equal to the growth rate of per-worker variables. 
Claim 2 and 3 deal with the related cases where either $g_{B_{F}}=0$ and $g_{B_{I}} \neq 0$ or $g_{B_{F}} \neq 0$ and $g_{B_{I}}=0$. They show that whenever $g_{B_{j}} \neq 0, j=F$, $I$, the respective aggregate must be Cobb-Douglas. Under this functional form technical change can be expressed as purely labor-augmenting at rate $g .{ }^{8}$ It is in this sense that the distinction between capital- and labor-augmenting technical change is blurred under a Cobb-Douglas.

In steady state the growth rates of $Y(t)$ and $I(t)$ must coincide. This requires an alignment in accordance with conditions (2.8) and (2.9), respectively. Intuitively, the growth rate of "labor-augmenting technical change" in the Cobb-Douglas aggregate must coincide with the growth rate of labor-augmenting technical change in the other aggregate. A remarkable feature of both conditions is then that $g_{B_{j}} \neq 0$ requires $g_{A_{F}} \neq g_{A_{I}}$. Hence, one may think of capital-augmenting technical change in the Cobb-Douglas aggregate as a necessary means to fill the gap between $g_{A_{F}}$ and $g_{A_{I}}$. Void of such a gap, there is no room for $B_{j}(t)$ to grow at a rate different from zero.

Claim 4 allows for $g_{B_{I}} \neq 0$ and $g_{B_{F}} \neq 0$. Accordingly, both aggregates must be Cobb-Douglas. Again, $g$, the growth rate of "labor-augmenting technical change" must be the same in both aggregates. Condition (2.10) states the required alignment.

Unlike Claim 2 and 3, a constellation involving $g_{A_{F}}=g_{A_{I}}$ is now consistent with a steady state if $g_{B_{F}}$ and $g_{B_{I}}$ adjust accordingly. Hence, the converse to Claim 1 is not true. Moreover, observe that $g_{A_{F}}=g_{A_{I}}$ and $g_{B_{I}}=g_{B_{F}}$ imply $\alpha_{F}=\alpha_{I}$.

Remark 1 Finally, it is worth mentioning that Corollary 2 also has some bearing on the cases where either $I(t)=0$, or $I(t)>0, B_{F}(t)=B_{I}(t), A_{F}(t)=A_{I}(t)$, and $I(t)=\beta F\left[B_{F}(t) K(t), A_{F}(t) L(t)\right]$, with $0<\beta<1$. The first of these cases concerns the scenario to which Uzawa's theorem directly applies. ${ }^{9}$ In the second case, aggregate investment has a functional form that coincides with the one of the aggregate production function up to a multiplicative constant. In both cases net output will be of the form

$$
V(t)=c_{V} F\left[B_{F}(t) K(t), A_{F}(t) L(t)\right], \quad c_{V}>0 .
$$

\footnotetext{
${ }^{8}$ In Theorem 1, I introduce $g$ as the growth rate of per-worker variables. In a slight abuse of notation, here I also use $g$ to denote the growth rate of "labor-augmenting" technical change in a Cobb-Douglas function. Observe that both growth rates coincide in steady state.

${ }^{9}$ See, e. g., Barro and Sala-í-Martin (2004), 78-80, for a discussion of this case.
} 
Hence, if $g_{B_{F}}=0$ then $g=g_{A_{F}}$. Moreover, if $g_{B_{F}} \neq 0$ then

$$
V(t)=\beta_{F} K(t)^{\alpha_{F}}\left(e^{g(t-\tau)} L(t)\right)^{1-\alpha_{F}}
$$

and $g=\alpha_{F} g_{B_{F}} /\left(1-\alpha_{F}\right)+g_{A_{F}}$.

\section{Endogenous Capital- and Labor-Augmenting Tech- nical Change - Three Examples}

Why is steady-state technical change purely labor-augmenting even in environments where capital-augmenting technical change is feasible? This section revisits three growth models with endogenous capital- and labor-augmenting technical change to shed light on this question. I show that the reduced form of these models satisfies either the assumptions of Theorem 1 or those of Uzawa's original theorem. As a consequence, steady-state technical change must have a representation as labor-augmenting. Moreover, unless Cobb-Douglas functions are involved capital-augmenting technical change vanishes in the steady state, and the economy's growth rate will be determined by labor-augmenting technical change alone.

Section 3.1 takes a new look at the competitive one-sector growth model developed in Irmen (2013). Here, $I(t)$ reflects aggregate productivity enhancing innovation investments and constitutes foregone output of the final good. This rightly suggests an application of Theorem 1. Section 3.2 revisits the R\&D-based variety expansion model of Acemoglu (2003). Here, scientists invent new varieties of differentiated intermediates. This model has no aggregate investments, i.e., $I(t)=0$, and Uzawa's original theorem characterizes the steady state. Section 3.3 studies an extension of Acemoglu (2003) that allows for price and market size effects to determine the direction of technical change (Acemoglu (2009), Chapter 15.6). In this model, some current final-good output is used to produce contemporaneous intermediate goods. Therefore, $I(t)>0$ and Theorem 1 is shown to inform us about the key features of the steady state. ${ }^{10}$

\footnotetext{
${ }^{10}$ All three models provide sophisticated micro-foundations that my discussion here must curtail for reasons of space. The interested reader is referred to the original contributions for details. The analysis in Irmen (2013) is set up in discrete time. Without loss of generality for my qualitative results, the following section presents the continuous-time version of this model.
} 


\subsection{Example 1: The One-Sector Model of Irmen (2013)}

At all $t$, the aggregate production function is $Y(t)=F[M(t), N(t)]$, where $Y(t)$ is output of the final good, $M(t)>0$ and $N(t)>0$ denote the total amount of tasks performed by either capital or labor. The function $F: \mathbb{R}_{+}^{2} \rightarrow \mathbb{R}_{+}$has constant returns to scale and is increasing in both arguments.

Let $k(t)=1 / B(t)$ denote the amount of capital required to perform each of the $M(t)$ tasks. Similarly, let $l(t)=1 / A(t)$ denote the amount of labor necessary to perform each of the $N(t)$ tasks. Accordingly, $B(t)>0$ and $A(t)>0$ indicate the productivity of capital and labor in the performance of their respective tasks. As before, $K(t)>0$ and $L(t)>0$ denote the capital and labor endowments. Then, full employment of capital and labor implies

$$
\begin{aligned}
M(t) k(t)=K(t) \quad & \Leftrightarrow \quad M(t)=B(t) K(t), \\
N(t) l(t)=L(t) \quad & \Leftrightarrow \quad N(t)=A(t) L(t) .
\end{aligned}
$$

Accordingly, aggregate production of the final good is equal to

$$
Y(t)=F[B(t) K(t), A(t) L(t)]
$$

and technical change represented by the evolution of $B(t)$ and $A(t)$ is capital- and labor-augmenting, respectively.

The economy can invest $M(t) i\left(q_{B}(t)\right)$ and $N(t) i\left(q_{A}(t)\right)$ units of contemporaneous output to increase $B(t)$ and $A(t)$ according to

$$
\dot{B}(t)=B(t)\left(q_{B}(t)-\delta_{B}\right) \quad \text { and } \quad \dot{A}(t)=A(t)\left(q_{A}(t)-\delta_{A}\right) .
$$

Here, $q_{B}(t)>0$ and $q_{A}(t)>0$ denote the growth rates of the respective productivity indicator gross of depreciation at rate $\delta_{B}>0$ and $\delta_{A}>0$, respectively. Moreover, $i: \mathbb{R}_{+} \rightarrow \mathbb{R}_{+}$specifies strictly positive investment requirements per task.

Using (3.1), the aggregate investments necessary to achieve strictly positive growth rates $q_{A}(t)$ and $q_{B}(t)$ are equal to $A(t) L(t) i\left(q_{A}(t)\right)$ and $B(t) K(t) i\left(q_{B}(t)\right)$, respectively. It follows that aggregate investment amounts to

$$
I(t)=A(t) L(t) i\left(q_{A}(t)\right)+B(t) K(t) i\left(q_{B}(t)\right) .
$$

Subtracting the latter from (3.2) delivers net output as

$$
V(t)=F[B(t) K(t), A(t) L(t)]-A(t) L(t) i\left(q_{A}(t)\right)-B(t) K(t) i\left(q_{B}(t)\right) .
$$


Proposition 1 Consider the economy described by (3.5), (2.4), (2.5), and (3.3). Suppose there exists a steady-state path starting at date $\tau<\infty$ such that $Y(t)>V(t)>C(t)>$ 0 for all $t \geq \tau$. Then, $q_{B}=\delta_{B}$,

$$
V(t)=F[B(\tau) K(t), A(t) L(t)]-A(t) L(t) i\left(q_{A}\right)-B(\tau) K(t) i\left(\delta_{B}\right),
$$

and $g=g_{A}=q_{A}-\delta_{A}$.

The proof of Proposition 1 also reveals the underlying intuition. Therefore, I develop it here. Start with the observation that $V(t)$ of (3.5) has constant returns to scale in $K(t)$ and $L(t)$. Hence, the economy described by (3.5), (2.4), and (2.5) has all features assumed in Theorem 1. Accordingly, steady-state technical change has a representation as labor-augmenting.

The definition of a steady state includes the requirement of exponential growth for $B(t)$ and $A(t)$, i.e., the accumulation equations (3.3) deliver $q^{B}(t)=q^{B}$ and $q^{A}(t)=q^{A}$. Now, define

$$
B_{F}(t) \equiv B(t), \quad A_{F}(t) \equiv A(t), \quad B_{I}(t) \equiv B(t) i\left(q^{B}\right), \quad A_{I}(t) \equiv A(t) i\left(q^{A}\right) .
$$

Then, in steady state (3.5) can be written as

$$
V(t)=F\left[B_{F}(t) K(t), A_{F}(t) L(t)\right]-\left(A_{I}(t) L(t)+B_{I}(t) K(t)\right),
$$

where the term in parenthesis is $I\left[B_{I}(t) K(t), A_{I}(t) L(t)\right]$. Hence, technical change is factor-augmenting in the net output function so that Corollary 2 applies. More precisely, since aggregate investment cannot be Cobb-Douglas I have to refer to either Claim 1 or to Claim 3. The definitions of $A_{F}(t)$ and $A_{I}(t)$ imply $g_{A_{F}}=$ $g_{A_{I}}=g_{A}$. Accordingly, (2.9) excludes $g_{B_{F}} \neq 0$. Finally, the definitions of $B_{F}(t)$ and $B_{I}(t)$ imply $g_{B_{F}}=g_{B_{I}}=g_{B}$. Since $g_{B_{F}}=0$ it follows that $g_{B_{F}}=g_{B_{I}}=0$ and $q_{B}=\delta_{B}$.

Hence, even though capital- and labor-augmenting technical change are endogenous, the properties of technical change in steady state are fully determined by Theorem 1 in conjunction with Corollary 2.

\subsection{Example 2: The Multi-Sector Model of Acemoglu (2003)}

Acemoglu's economy comprises four sectors on the production side and a representative consumer. It is therefore appropriate to split the discussion into three 
parts. Section 3.2.1 starts with a description of the economy's production side. Moreover, I derive some important relations that arise for symmetric (equilibrium) configurations. Section 3.2.2 presents the relevant aspects of the household side that lead to the conclusion that gross and net output coincide. Section 3.2.3 looks at the steady-state path and establishes the link to Uzawa's original theorem. Time is continuous. All prices are expressed in units of the contemporaneous final-good.

\subsubsection{Production and Research}

First, there is a competitive sector producing the final good, $Y(t)$, out of a capitalintensive intermediate good, $Y_{K}(t)$, and a labor-intensive intermediate good, $Y_{L}(t)$. The production function of the final good sector is ${ }^{11}$

$$
Y(t)=F\left[Y_{K}(t), Y_{L}(t)\right]
$$

It exhibits constant returns to scale and positive but diminishing marginal products. Profit maximization delivers

$$
F_{1}\left[Y_{K}(t), Y_{L}(t)\right]=p_{K}(t) \text { and } \quad F_{2}\left[Y_{K}(t), Y_{L}(t)\right]=p_{L}(t)
$$

where $p_{K}(t)$ and $p_{L}(t)$ denote the prices of the respective intermediate at $t$.

Second, there is a competitive intermediate-good sector that produces $Y_{K}(t)$ and $Y_{L}(t)$ out of differentiated intermediates. The respective production functions are of the CES-type,

$$
Y_{K}(t)=\left[\int_{0}^{m(t)} \sqrt{y_{k}(i, t)} d i\right]^{2} \text { and } Y_{L}(t)=\left[\int_{0}^{n(t)} \sqrt{y_{l}(i, t)} d i\right]^{2} .
$$

Here, $[0, m(t)]$ and $[0, n(t)]$ denote disjoint sets of intermediates available at $t$. All intermediates in use at $t$ fully depreciate afterwards. Therefore, profit maximization in this intermediate-good sector is static and delivers the demand for each

\footnotetext{
${ }^{11}$ Acemoglu assumes $F$ to be a CES production function. This specification is not necessary for my purpose here (though, the elasticity of substitution plays an important role in Acemoglu's analysis). When referring to other functional forms that appear in Acemoglu (2003), I use particular values for the following parameters: $\beta=1 / 2, b_{k}=b_{l}=1$. Moreover, I set the function $\phi(s)=1$, for $s=S_{k}, S_{l}$. These choices simplify the exposition but are without loss of generality for the qualitative results I derive.
} 
intermediate as

$$
y_{k}(i, t)=\left(\frac{p_{K}(t)}{p_{k}(i, t)}\right)^{2} Y_{K}(t) \quad \text { and } \quad y_{l}(i, t)=\left(\frac{p_{L}(t)}{p_{l}(i, t)}\right)^{2} Y_{L}(t)
$$

where $p_{k}(i, t)$ is the price per unit of $y_{k}(i, t)$ and $p_{l}(i, t)$ is the one per unit of $y_{l}(i, t)$.

The third sector comprises monopolists each producing one of the intermediate goods with a linear production function, i. e., $y_{k}(i, t)=k(i, t)$ and $y_{l}(i, t)=l(i, t)$, where $k(i, t)$ is capital input and $l(i, t)$ the input of unskilled labor at $t$. Here, profit-maximization leads to (symmetric) prices

$$
p_{k}(t)=2 r(t) \quad \text { and } \quad p_{l}(t)=2 w(t),
$$

where $r(t)$ and $w(t)$ denote the price per unit of capital and labor that the monopolists incur.

Consider a symmetric configuration of these three sectors. Then, $y_{k}(t)=k(t)$, $y_{l}(t)=l(t)$, and the factor market clearing conditions read $m(t) k(t)=K(t)$ and $n(t) l(t)=L$, respectively. Here, $K(t)>0$ and $L>0$ denote the endowments of capital and unskilled labor. ${ }^{12}$ As a consequence, $Y_{K}(t)=m(t) K(t)$ and $Y_{L}(t)=$ $n(t) L$, so that

$$
Y(t)=F(m(t) K(t), n(t) L) .
$$

Hence, increasing $m(t)$ and $n(t)$ has an interpretation as capital-, respectively, labor-augmenting technical change. The flow profits accruing to each of the respective monopolist amount to

$$
\pi_{k}(t)=\frac{r(t) K(t)}{m(t)} \quad \text { and } \quad \pi_{l}(t)=\frac{w(t) L}{n(t)} .
$$

The fourth sector is the research sector. It consists of competitive research firms that invent new varieties of intermediates. Let $S_{k}(t) \geq 0\left(S_{l}(t) \geq 0\right)$ denote the "number" of scientists that research firms engaged in the invention of new capital-intensive (labor-intensive) intermediates hire. These research firms have access to the following technologies for invention

$$
\frac{\dot{m}(t)}{m(t)}=S_{k}(t)-\delta \quad \text { and } \quad \frac{\dot{n}(t)}{n(t)}=S_{l}(t)-\delta,
$$

\footnotetext{
${ }^{12}$ To simplify the notation, capital does not depreciate. Moreover, I follow Acemoglu and set $L(t)=L$.
} 
where $\delta \in \mathbb{R}_{++}$is the obsolescence rate of existing varieties. At all $t$, there are $S$ scientists in the economy, i.e., market clearing requires $S_{k}(t)+S_{l}(t)=S$. Let $\omega_{S}(t)$ denote the real wage of a scientist at $t$. Competition in the research sector implies zero profits, i. e., $\omega_{S}(t)$ must be equal to the value a scientist creates by inventing new varieties. Denote $V_{k}(t)$ and $V_{l}(t)$ the value of a variety of the respective type at $t$. Then, using (3.13) any configuration where research is conducted on both types of intermediates must have

$$
\omega_{S}(t)=m(t) V_{k}(t)=n(t) V_{l}(t) .
$$

\subsubsection{The Household Side}

The economy admits a representative household. The economy's net output corresponds to the resources that this household can allocate to consumption and to the accumulation of capital. To determine this amount the household's flow income must be derived.

The household owns the capital stock, $K(t), L$ units of unskilled labor, $n(t)$ and $m(t)$ monopoly firms each producing one intermediate-good variety, all research firms, and $S$ units of labor services as a scientist. Potentially, it earns income on all these possessions.

Using (3.7) - (3.10) and the respective factor market clearing conditions reveals that

$$
r(t)=\frac{m(t) F_{1}[m(t) K(t), n(t) L]}{2} \quad \text { and } \quad w(t)=\frac{n(t) F_{2}[m(t) K(t), n(t) L]}{2},
$$

i. e., due to the price-cost margins of the monopolists the prices of capital and of unskilled labor fall short of their respective marginal product in the production of the final good.

With constant returns in (3.6) it follows that the household income from capital holdings and unskilled labor amounts to

$$
r(t) K(t)+w(t) L=\frac{Y(t)}{2} .
$$

Invoking (3.12) the dividend income of the household resulting from the ownership of intermediate-good monopolists is $m(t) \pi_{k}(t)+n(t) \pi_{l}(t)$. With (3.15) one finds

$$
m(t) \pi_{k}(t)+n(t) \pi_{l}(t)=\frac{Y(t)}{2} .
$$


Inspection of (3.15) and (3.16) reveals that household income due to the ownership of the four factors of production in (3.11) adds up to $Y(t)$.

What about the wage income of the scientists? In a sense, the representative household pays it to itself. Since research firms make zero profits, their revenue from selling blueprints satisfies $m(t) V_{k}(t) S_{k}(t)+n(t) V_{l}(t) S_{l}(t)=w_{S} S$. New monopolists buy these blueprints at a price equal to $V_{k}(t)$ and $V_{l}(t)$, respectively, and finance these purchases through the issue of shares. These shares are bought by the representative household and yield dividends equal to $\pi_{k}(t)$ and $\pi_{l}(t)$ in the future. Hence, this purchase of shares may be interpreted an act of saving. At the same time, it qualifies as an innovation investment since the resources are eventually transferred to the research firms and allow them to break even. However, the important point is that these resources correspond to the scientists' aggregate salary. Therefore, they remain available as household income. In other words, since innovation investments generate income in the present model they leave the amount of resources available for consumption and capital accumulation unchanged. Accordingly, net output and gross output coincide, i. e., $V(t)=Y(t)$.

\subsubsection{Net Output and the Steady State}

The following proposition highlights the link between the steady-state path of the economy and Theorem 1.

Proposition 2 Consider the economy described by (3.11), (2.4), (2.5) with $g_{L}=0$, and (3.13). Suppose there exists a steady-state path starting at date $\tau<\infty$ such that $Y(t)>C(t)>0$ for all $t \geq \tau$. Then, the following holds:

1. If $g_{m} \neq 0$, then net output has the form

$$
V(t)=\beta_{F}[K(t)]^{\alpha_{F}}\left[e^{g(t-\tau)} L\right]^{1-\alpha_{F}},
$$

where

$$
\begin{gathered}
g=\frac{\alpha_{F} g_{m}}{1-\alpha_{F}}+g_{n} \\
g_{m}=S_{k}-\delta, \quad g_{n}=S_{l}-\delta, \quad \text { and } S_{k}+S_{l}=S .
\end{gathered}
$$

2. If $F$ is not Cobb-Douglas then $g_{m}=0$ and $g=g_{n}=S-2 \delta$. 
To prove Proposition 2 start with the observation that $F$ of (3.11) has constant returns to capital and labor. The conclusion of Section 3.2.2 is that $V(t)=Y(t)$. Hence, the economy described by (3.11), (2.4), and (2.5) with $g_{L}=0$ satisfies all assumptions of Theorem 1 for $I(t)=0$, i. e., of Uzawa's original theorem. Accordingly, steady-state technical change has a representation as labor-augmenting.

The definition of a steady state includes the requirement of exponential growth for $m(t)$ and $n(t)$. Hence, with (3.13) it must be that $g_{m}=S_{k}-\delta$ and $g_{n}=S_{l}-\delta$. Let $B_{F}(t) \equiv m(t)$ and $A_{F}(t) \equiv n(t)$. Then, (net) output can be written as $V(t)=$ $F\left[B_{F}(t) K(t), A_{F}(t) L\right]$. Accordingly, Remark 1 applies and suggests the distinction of the two cases mentioned in the proposition. First, $g_{m} \neq 0$ can only occur if $F$ is of the Cobb-Douglas type. Here, the parameters $\alpha_{F}$ and $\beta_{F}$ are as introduced in Corollary 2. Accordingly, $g=\alpha_{F} g_{m} /\left(1-\alpha_{F}\right)+g_{n}$ is the steady-state growth rate of the economy. The steady-state levels of $g_{m}$ and $g_{n}$ remain undetermined but will have to be consistent with the market clearing condition for scientists, $S_{k}+S_{l}=S$.

The second case follows immediately from the first. Any functional form other than the Cobb-Douglas type implies $g_{m}=0$ and, under full employment of scientists, $g=g_{n}=S-2 \delta$.

Hence, Theorem 1 dictates the feasible form of steady-state technical change. However, as $I(t)=0$ and technical change is factor-augmenting Corollary 2 can be replaced by the simpler reasoning of Remark 1 . The following extension of Acemoglu (2003) reveals that a similar simplification may be possible even if $I(t)>0$.

\subsection{Example 3: The Multi-Sector Model of Acemoglu (2009)}

For the purpose of this paper it is also revealing to take a new look at the extension of the model in Acemoglu (2003) that allows for a price effect and a market size effect to determine the direction of technical change (see, Acemoglu (2009), Chapter 15.6). Here, I show that this extension gives rise to a strictly positive aggregate investment function, i. e., $I(t)>0$, that suggests an application of Theorem 1. This is the main point of Proposition 3 below.

To account for a price and a market size effect two changes to the production sector of Section 3.2.1 are made. First, scale effects are added to the production functions in (3.8). Second, monopolists manufacture machines (intermediate goods) 
out of current final-good output as the only input. Due to the latter change, gross and net output differ.

To make this more precise reconsider the second sector of Section 3.2.1, i. e., the competitive intermediate-good firms that produce $Y_{K}(t)$ and $Y_{L}(t)$, respectively. Replace the production functions in (3.8) by

$$
Y_{K}(t)=2 \sqrt{K(t)} \int_{0}^{m(t)} \sqrt{y_{k}(i, t)} d i \text { and } Y_{L}(t)=2 \sqrt{L} \int_{0}^{n(t)} \sqrt{y_{l}(i, t)} d i .
$$

Here, the appearance of $K(t)$ and $L$ is new. Hence, the disjoint sets $[0, m(t)]$ and $[0, n(t)]$ represent machines that are either complementary to capital or to labor. All machines in use at $t$ fully depreciate after use. Therefore, profit maximization in this intermediate-good sector is static and delivers the demand for each machine as

$$
y_{k}(i, t)=\left(\frac{p_{K}(t)}{p_{k}(i, t)}\right)^{2} K(t) \quad \text { and } \quad y_{l}(i, t)=\left(\frac{p_{L}(t)}{p_{l}(i, t)}\right)^{2} L .
$$

Moreover, the demands for capital and labor satisfy the first-order conditions

$$
\frac{p_{K}(t) \int_{0}^{m(t)} \sqrt{y_{k}(i, t)} d i}{\sqrt{K(t)}}=r(t) \text { and } \frac{p_{L}(t) \int_{0}^{n(t)} \sqrt{y_{l}(i, t)} d i}{\sqrt{L}}=w(t) .
$$

Next, reconsider the third sector of Section 3.2.1, i. e., the monopolists each producing one variety of the machines used in (3.17). The new feature is that the production of machines uses current final output as the sole input. Assume that $1 / 2$ units of the final good are used as an input per machine. Then, profit maximization of all monopolists delivers (symmetric) machine prices

$$
p_{k}(t)=p_{l}(t)=1,
$$

and monopolistic flow profits are equal to

$$
\pi_{k}(t)=\frac{p_{K}^{2}(t) K(t)}{2} \text { and } \pi_{l}(t)=\frac{p_{L}^{2}(t) L}{2} .
$$

With these changes a symmetric configuration of the three production sectors has the following important properties. Combining (3.17), (3.18), and (3.20) delivers the derived production functions for the two intermediates, i. e.,

$$
Y_{K}(t)=2 p_{K}(t) m(t) K(t) \quad \text { and } \quad Y_{L}(t)=2 p_{L}(t) n(t) L(t)
$$


With (3.6) final-good output may be written as

$$
Y(t)=2 F\left(p_{K}(t) m(t) K(t), p_{L}(t) n(t) L\right) .
$$

Hence, increasing $p_{K}(t) m(t)$ and $p_{L}(t) n(t)$ has an interpretation as capital-, respectively, labor-augmenting technical change. In addition, with (3.19) factor prices are

$$
m(t) p_{K}^{2}(t)=r(t) \quad \text { and } \quad n(t) p_{L}^{2}(t)=w(t) .
$$

What are the relevant implications for the household sector? As in the previous section, the representative household owns the capital stock, $K(t), L$ units of unskilled labor, $n(t)$ and $m(t)$ monopoly firms producing one intermediate good each, all research firms, and $S$ units of labor services as a scientist. Potentially, it earns income on all these possessions.

From (3.17), (3.18), (3.20), (3.24) and constant returns in (3.6) it follows that the household income from capital holdings and unskilled labor amounts to

$$
r(t) K(t)+w(t) L=\frac{Y(t)}{2} .
$$

Invoking (3.21), (3.24), and (3.25) the dividend income of the household resulting from the ownership of intermediate-good monopolists is

$$
m(t) \pi_{k}(t)+n(t) \pi_{l}(t)=\frac{r(t) K(t)+w(t) L}{2}=\frac{Y(t)}{4} .
$$

Inspection of (3.25) and (3.26) reveals that household income due to the ownership of the four factors of production in (3.23) adds up to $3 Y(t) / 4$. The remaining quarter corresponds to the final output used as an input in the production of the $y_{k}(i, t)$ and $y_{l}(i, t)$ machines. Let $I(t)$ denote the total amount of these resources. Since each machine is manufactured out of $1 / 2$ units of the final good one obtains for a symmetric configuration

$$
I(t)=\frac{m(t) y_{k}(t)+n(t) y_{l}(t)}{2}=\frac{r(t) K(t)+w(t) L}{2}=\frac{Y(t)}{4},
$$

where use is made of (3.18), (3.20), (3.24), and (3.25). For the reasons set out in Section 3.2.2, the research sector does not add any net output that can be either consumed or used to accumulate capital. Hence, net output is equal to

$$
V(t)=Y(t)-I(t) .
$$


With (3.23), one finds

$$
V(t)=\frac{3 Y(t)}{4}=\frac{3 F\left(p_{K}(t) m(t) K(t), p_{L}(t) n(t) L\right)}{2} .
$$

The following proposition has the link between the steady-state path of the economy and Theorem 1 . To simplify the notation define $B_{F}(t) \equiv p_{K}(t) m(t), A_{F}(t) \equiv$ $p_{L}(t) n(t), g_{B_{F}} \equiv g_{p_{K}}+g_{m}$, and $g_{A_{F}} \equiv g_{p_{L}}+g_{n}$.

Proposition 3 Consider the economy described by (3.29), (2.4), (2.5) with $g_{L}=0$, and (3.13). Suppose there exists a steady-state path starting at date $\tau<\infty$ such that $Y(t)>V(t)>C(t)>0$ for all $t \geq \tau$. Then, the following holds:

1. If $g_{B_{F}} \neq 0$, then net output has the form

$$
V(t)=\beta_{F}[K(t)]^{\alpha_{F}}\left[e^{g(t-\tau)} L\right]^{1-\alpha_{F}},
$$

where

$$
\begin{gathered}
g=\frac{\alpha_{F} g_{B_{F}}}{1-\alpha_{F}}+g_{A_{F}} \\
g_{B_{F}}=S_{k}-\delta-g_{p_{K^{\prime}}}, \quad g_{A_{F}}=S_{l}-\delta-g_{p_{L}}, \quad \text { and } \quad S_{k}+S_{l}=S .
\end{gathered}
$$

2. If $F$ is not Cobb-Douglas, then $g_{B_{F}}=0$ and $g=g_{A_{F}}=S-2 \delta-\left(g_{p_{K}}+g_{p_{L}}\right)$.

Despite the more complex structure of the economy, Proposition 3 looks strikingly similar to Proposition 2. To see why this is so start with the observation that $F$ of (3.29) has constant returns to capital and labor. Since $V(t)=Y(t)-I(t)$ and $I(t)=Y(t) / 4>0$, the economy described by (3.29), (2.4), and (2.5) with $g_{L}=0$ satisfies the assumptions of Theorem 1 with $I(t)>0$. Accordingly, in steady state, technical change has a representation as purely labor-augmenting. The technologies for invention stated in (3.13) are consistent with this requirement.

From (3.29) it also follows that the aggregate production and the aggregate investment function are the same up to a multiplicative constant. Moreover, technical change is factor-augmenting. Hence, if $g_{B_{F}} \neq 0$ then Remark 1 implies that net 
output must be Cobb-Douglas with a growth rate of "labor-augmenting technical change" as indicated in Claim 1 of Proposition 3. ${ }^{13}$ As in Proposition 2 the growth rates of $B_{F}(t)$ and $A_{F}(t)$ remain undetermined. Moreover, whenever $F$ is not Cobb-Douglas, $B_{F}(t)$ is constant in steady state. ${ }^{14}$

\section{Concluding Remarks}

Why is technical change labor-augmenting in the steady state? This paper argues that the answer to this question hinges on whether the reduced form of the macroeconomic model under scrutiny is consistent with the generalized steadystate growth theorem established above as Theorem 1 . If this is the case then the answer is clear: in steady state technical change must be labor-augmenting in the aggregate production function, the aggregate investment function, and, hence, in the net output function. Moreover, the growth rate of per-worker variables is determined by the growth rate of labor-augmenting technical change.

Capital-augmenting technical change may only arise in steady state if technical change retains a representation as labor-augmenting in the net output function. This is feasible when technical change is factor-augmenting, and the aggregate production function and/or the aggregate investment function are of the CobbDouglas type. In any of these cases, the growth rates of the capital- and the laboraugmenting technologies in these aggregates must be aligned so that these aggregates grow at the same rate.

From the perspective of these findings, the present paper revisits three recent growth models with endogenous capital- and labor-augmenting technical change. In Irmen (2013), current output serves as an input in productivity enhancing innovation investments. These innovation investments reduce the amount of

\footnotetext{
${ }^{13}$ To justify Claim 1 of Proposition 3, one may alternatively invoke Claim 4 of Corollary 2 with $\alpha_{F}=\alpha_{I}$.

${ }^{14}$ Observe that the way I defined factor-augmenting technical change gives rise to the growth rates $g_{p_{K}}$ and $g_{p_{L}}$ to show up in Proposition 3. Generically, these growth rates vanish in steady state. For instance, if the aggregate production function $F$ of (2.1) has a constant elasticity of substitution different from unity then the factor-price frontier implied by the first-order conditions (3.7) requires $g_{p_{K}}=g_{p_{L}}=0$ in steady state. However, this reasoning is not directly related to Theorem 1 and, therefore, will not be deepened here.
} 
resources available for consumption and capital accumulation, i.e., the aggregate investment function is strictly positive. Therefore, Theorem 1 applies to this model. Moreover, in conjunction with Corollary 2 the pattern of steady-state technical change can be inferred.

In Acemoglu (2003) innovations occur in a research sector that employs scientists. The resources necessary to finance research tend to reduce what is left for consumption and capital accumulation. However, these resources accrue as income to scientists and, therefore, remain available for consumption and capital accumulation. In other words, gross and net output coincide. As the latter exhibits constant returns in capital and (unskilled) labor Uzawa's original theorem can be applied to derive the pattern of technical change in steady state.

Finally, I study an extension of Acemoglu (2003) that accounts for market-size effects (Acemoglu (2009), Chapter 15.6). This model gives rise to a strictly positive aggregate investment function since contemporaneous final-good output serves as an input for downstream intermediate-good monopolists. As a consequence, gross and net output differ, and Theorem 1 applies. Since, up to a multiplicative constant, the aggregate production function is the same as the aggregate investment function the analysis of the steady-state pattern of technical change leads to similar conclusions as for Acemoglu (2003).

Hence, in the existing literature the generalized steady-state growth theorem provides the intuitive underpinning for why only labor-augmenting technical change is viable in the steady state even though capital-augmenting technical change is feasible. However, this observation should not be interpreted as a plea to neglect or eliminate capital-augmenting technical change altogether. Indeed, the results derived in Irmen (2013) suggest that both the normative and the positive implications of models with endogenous technical change crucially hinge upon whether capital-augmenting technical change is included in the analysis or not. Future research will have to elucidate this point. It will be of particular interest to study whether policy recommendations are robust if endogenous capital-augmenting technical change is added to the picture. 


\section{Appendix: Proofs}

\subsection{Proof of Theorem 1}

Observe that $Y(t)>V(t)>C(t)>0$ implies both $Y(t)>I(t)>0$ and $V(t)>C(t)>0$. Moreover, without loss of generality let $\tau=0$.

Part I Given time-invariant growth rates, date $t$ quantities may be expressed in terms of date 0 quantities, i. e., $V(t)=V(0) e^{g V t}, Y(t)=Y(0) e^{g \gamma t}$, and $I(t)=I(0) e^{g_{I} t}$. Then, from the definition of $V(t)$, I have for all $t \geq 0$

$$
V(0) e^{g_{V} t}=Y(0) e^{g Y t}-I(0) e^{g_{I} t} .
$$

Dividing both sides by $e^{g_{V} t}$ gives

$$
V(0)=Y(0) e^{\left(g_{Y}-g_{V}\right) t}-I(0) e^{\left(g_{I}-g_{V}\right) t} .
$$

Differentiation with respect to $t$ delivers

$$
0=\left(g_{Y}-g_{V}\right) Y(0) e^{\left(g_{Y}-g_{V}\right) t}-\left(g_{I}-g_{V}\right) I(0) e^{\left(g_{I}-g_{V}\right) t} .
$$

The latter can hold for all $t$ if any of the following conditions are satisfied; a) $g_{V}=g_{Y}=g_{I}, \mathrm{~b}$ ) $g_{Y}=g_{I}$ and $Y(0)=I(0)$, c) $g_{V}=g_{Y}$ and $I(0)=0$, and d) $g_{V}=g_{I}$ and $Y(0)=0$. Alternatives b) - d) contradict $Y(0)>I(0)>0$. Hence, $g_{V}=g_{Y}=g_{I}$ must apply.

With $K(t)=K(0) e^{g K t}$ and $C(t)=C(0) e^{g} C^{t}$, capital accumulation of (2.4) delivers

$$
K(0) e^{g_{K} t}\left(g_{K}+\delta_{K}\right)=V(0) e^{g_{V} t}-C(0) e^{g_{C} t} .
$$

Divide both sides by $e^{g_{K} t}$ and obtain

$$
K(0)\left(g_{K}+\delta_{K}\right)=V(0) e^{\left(g_{V}-g_{K}\right) t}-C(0) e^{\left(g_{C}-g_{K}\right) t} .
$$

Differentiation of the latter with respect to $t$ gives

$$
0=\left(g_{V}-g_{K}\right) V(0) e^{\left(g_{V}-g_{K}\right) t}-\left(g_{C}-g_{K}\right) C(0) e^{\left(g_{C}-g_{K}\right) t} .
$$

The latter can hold for all $t$ if any of the following conditions are satisfied: a) $g_{V}=g_{K}=g_{C}$, b) $g_{V}=g_{C}$ and $V(0)=C(0)$, c) $g_{V}=g_{K}$ and $C(0)=0$, and d) $g_{C}=g_{K}$ and $V(0)=0$. Alternatives b) - d) contradict $V(0)>C(0)>0$. Hence, $g_{V}=g_{K}=g_{C}$ must apply as claimed. This completes the proof of Part I.

Part II In light of (2.3), for any $t \geq 0$, net output at time 0 may be written as

$$
e^{-g_{V} t} \cdot V(t)=\tilde{V}\left[e^{-g_{K} t} \cdot K(t), e^{-g_{L} t} \cdot L(t), \mathbf{A}_{F}(0), \mathbf{A}_{I}(0)\right] .
$$

Multiplying both sides by $e^{g_{V} t}$ and using constant returns of $\tilde{V}$ gives

$$
V(t)=\tilde{V}\left[e^{\left(g_{V}-g_{K}\right) t} \cdot K(t), e^{\left(g_{V}-g_{L}\right) t} \cdot L(t), \mathbf{A}_{F}(0), \mathbf{A}_{I}(0)\right] .
$$


From Part I, I have $g_{V}=g_{K}$, hence for any $t \geq 0$

$$
V(t)=\tilde{V}\left[K(t), e^{\left(g_{V}-g_{L}\right) t} \cdot L(t), \mathbf{A}_{F}(0), \mathbf{A}_{I}(0)\right] .
$$

Since the latter equation is true for all $t \geq 0$ and $\tilde{V}$ is linear homogenous in the first two arguments, there exists a linear homogeneous function $V: \mathbb{R}_{+}^{2} \rightarrow \mathbb{R}_{+}$such that

$$
V(t)=V\left[K(t), e^{\left(g_{V}-g_{L}\right) t} \cdot L(t)\right]=V[K(t), A(t) L(t)]
$$

with $A(t)=e^{\left(g_{V}-g_{L}\right) t} \in \mathbb{R}_{++}$.

Part I and constant returns to scale imply that net output per-worker as well as all other perworker variables grow at rate $g=g_{V}-g_{L}$. This establishes the second part of the theorem.

\subsection{Proof of Corollary 1}

Again, without loss of generality let $\tau=0$.

Part I Since $Y(t)>V(t)=C(t)>0$ we now have $Y(t)>I(t)>0$ and $V(t)=C(t)>0$. Therefore, the proof of $g_{V}=g_{Y}=g_{I}$ remains as in the proof of Theorem 1. However, $V(t)=C(t)$ and (2.4) deliver $g_{V}=g_{C}$ and $g_{K}=-\delta_{K}$, respectively. This proves Part I of Corollary 1.

Part II The first two steps in the proof of Part II of Theorem 1 remain valid. Then, using $g_{K}=-\delta_{K}$ in (5.3) delivers for any $t \geq 0$

$$
V(t)=\tilde{V}\left[e^{\left(g_{V}+\delta\right) t} \cdot K(t), e^{\left(g_{V}-g_{L}\right) t} \cdot L(t), \mathbf{A}_{F}(0), \mathbf{A}_{I}(0)\right] .
$$

Since the latter equation is true for all $t \geq 0$ and $\tilde{V}$ is linear homogenous in the first two arguments, there exists a linear homogeneous function $V: \mathbb{R}_{+}^{2} \rightarrow \mathbb{R}_{+}$such that

$$
V(t)=V\left[e^{\left(g_{V}+\delta_{K}\right) t} \cdot K(t), e^{\left(g_{V}-g_{L}\right) t} \cdot L(t)\right]=V[B(t) K(t), A(t) L(t)]
$$

with $B(t)=e^{\left(g_{V}+\delta_{K}\right) t} \in \mathbb{R}_{++}$and $A(t)=e^{\left(g_{V}-g_{L}\right) t} \in \mathbb{R}_{++}$. Hence, $V(t)$ is as stated in Corollary 1 . Capital per worker, $K(t) / L(t)$, grows at rate $g_{K}-g_{L}=-\left(\delta_{K}+g_{L}\right)$. Moreover, since $g_{V}=g_{Y}=$ $g_{I}=g_{C}$ all remaining per-worker variables grow at rate $g=g_{V}-g_{L}$.

\subsection{Proof of Corollary 2}

Let me introduce

$$
\kappa_{F}(t)=\frac{A_{F}(t) L(t)}{B_{F}(t) K(t)} \quad \text { and } \quad \kappa_{I}(t)=\frac{A_{I}(t) L(t)}{B_{I}(t) K(t)} .
$$


Now, consider (2.7) and divide by $K(t)$. This gives

$$
\begin{aligned}
\frac{V(t)}{K(t)} & =\frac{Y(t)}{K(t)}-\frac{I(t)}{K(t)} \\
& =B_{F}(t) F\left[1, \kappa_{F}(t)\right]-B_{I}(t) I\left[1, \kappa_{I}(t)\right], \\
& =B_{F}(t) f\left(\kappa_{F}(t)\right)-B_{I}(t) i\left(\kappa_{I}(t)\right),
\end{aligned}
$$

where $f: \mathbb{R}_{+} \rightarrow \mathbb{R}_{+}$and $i: \mathbb{R}_{+}^{2} \rightarrow \mathbb{R}_{+}$are respectively defined as $f\left(\kappa_{F}(t)\right) \equiv F\left[1, \kappa_{F}(t)\right]$ and $i\left(\kappa_{I}(t)\right) \equiv I\left[1, \kappa_{I}(t)\right]$.

Observe that net output of (2.7) is a special case of the formulation given in (2.3). Therefore, Part I of Theorem 1 applies and imposes the requirement that $g_{V}=g_{Y}=g_{I}=g_{K}$, i. e., the fraction $V(t) / K(t)$ as well as $Y(t) / K(t)=B_{F}(t) f\left(\kappa_{F}(t)\right)$ and $I(t) / K(t)=B_{I}(t) i\left(\kappa_{I}(t)\right)$ are constant in steady state. Then, four cases may arise. They correspond to the four claims made in the corollary. I consider each in turn.

1. If $g_{B_{F}}=g_{B_{I}}=0$ then $g_{\kappa_{F}}=g_{\kappa_{I}}=0$. Taken together, the implication is that $g_{A_{F}}=g_{A_{I}}=$ $g_{K}-g_{L}$. Since $g_{V}=g_{K}$, the growth rate of per-worker variables is $g_{A_{F}}=g_{A_{I}}=g_{V}-g_{L}=$ $g$.

2. If $g_{B_{F}}=0$ and $g_{B_{I}} \neq 0$ then $g_{\kappa_{F}}=0$ whereas $g_{\kappa_{I}} \neq 0$. For aggregate production the implication is that $g_{A_{F}}=g_{K}-g_{L}$. For aggregate investment the growth rates $g_{B_{I}} \neq 0$ and $g_{\kappa_{I}} \neq 0$ must be of opposite sign such that $B_{I}(t) i\left(\kappa_{I}(t)\right)$ with $i^{\prime}\left(\kappa_{I}(t)\right)>0$ can remain constant over time. In other words, the time derivative of this product must vanish, i. e.,

$$
\dot{B}_{I}(t) i\left(\kappa_{I}(t)\right)+B_{I}(t) \dot{\kappa}_{I}(t) i^{\prime}\left(\kappa_{I}(t)\right)=0,
$$

or, in steady state,

$$
\frac{i^{\prime}\left(\kappa_{I}(t)\right) \kappa_{I}(t)}{i\left(\kappa_{I}(t)\right)}=-\frac{g_{B_{I}}}{g_{\kappa_{I}}} .
$$

Integration reveals that the solution can be written as

$$
i\left(\kappa_{I}(t)\right)=c_{I} \kappa_{I}(t)^{1-\alpha_{I}},
$$

where $c_{I}>0$ is a constant of integration and $\alpha_{I}=1+g_{B_{I}} / g_{\kappa_{I}}$. A positive, yet declining marginal product of capital requires $0<\alpha_{I}<1$. Then, for all $t \geq \tau$

$$
I(t)=c_{I}\left(B_{I}(t) K(t)\right)^{\alpha_{I}}\left(A_{I}(t) L(t)\right)^{1-\alpha_{I}}=c_{I}\left(B_{I}(\tau) K(t)\right)^{\alpha_{I}}\left(A_{I}(\tau) e^{g(t-\tau)} L(t)\right)^{1-\alpha_{I}},
$$

where $g=\alpha_{I} g_{B_{I}} /\left(1-\alpha_{I}\right)+g_{A_{I}}$. Introducing the constant $\beta_{I}, I(t)$ may be written as stated in Claim 2.

To align $g_{I}$ and $g_{Y}$, express the growth rate of $I(t)$ as

$$
g_{I}=\alpha_{I}\left(g_{B_{I}}+g_{K}\right)+\left(1-\alpha_{I}\right)\left(g_{A_{I}}+g_{L}\right) .
$$


Since $g_{I}=g_{K}$ the latter becomes

$$
g_{I}=\frac{\alpha_{I} g_{B_{I}}}{1-\alpha_{I}}+g_{A_{I}}+g_{L}
$$

Next, recall that $g_{Y}=g_{K}=g_{A_{F}}+g_{L}$. Then, $g_{Y}=g_{I}$ requires

$$
g_{A_{F}}=\frac{\alpha_{I} g_{B_{I}}}{1-\alpha_{I}}+g_{A_{I}}=g \text {. }
$$

Hence, any set of growth rates $\left\{g_{A_{F}}, g_{B_{I}}, g_{A_{I}}\right\} \in \mathbb{R}^{2} \times \mathbb{R} \backslash\{0\}$ must satisfy the latter condition to be consistent with a steady state.

3. The case where $g_{B_{F}} \neq 0$ and $g_{B_{I}}=0$ is the mirror image of the previous case. Mutatis mutandis, the proof of Claim 2 applies here, too.

4. If $g_{B_{F}} \neq 0$ and $g_{B_{I}} \neq 0$ then the proof of Cases 2 and 3 implies immediately that aggregate production and aggregate investment can be written as

$$
\begin{aligned}
& Y(t)=\beta_{F} K(t)^{\alpha_{F}}\left(e^{g(t-\tau)} L(t)\right)^{1-\alpha_{F}}, \\
& I(t)=\beta_{I} K(t)^{\alpha_{I}}\left(e^{g(t-\tau)} L(t)\right)^{1-\alpha_{I}},
\end{aligned}
$$

where $g$ is given by (2.10), the condition that any set of growth rates $\left\{g_{B_{F}}, g_{A_{F}}, g_{B_{I}}, g_{A_{I}}\right\} \in$ $\mathbb{R}^{2} \times \mathbb{R} \backslash\{0,0\}$ must satisfy to be consistent with a steady state. Accordingly, net output has the form given in Claim 4 .

\subsection{Proof of Proposition 1, 2, and 3}

To be found in the main text. 


\section{References}

ACEmoglu, D. (2003): “Labor- and Capital-Augmenting Technical Change," Journal of European Economic Association, 1(1), 1-37.

(2009): Introduction to Modern Economic Growth. Princeton University Press, Princeton, New Jersey.

BARRO, R. J., AND X. SAlA-Í-MARTIN (2004): Economic Growth. MIT Press, Cambridge, MA, 2nd edn.

IRMEN, A. (2011): "Steady-State Growth and the Elasticity of Substitution," Journal of Economic Dynamics and Control, 35(8), 1215-1228.

(2013): “Capital- and Labor-Saving Technical Change in an Aging Economy," CREA Discussion Paper Series 2013-27, Center for Research in Economic Analysis, University of Luxembourg.

JONES, C. I. (2005): “The Shape of Production Functions and the Direction of Technical Change," The Quarterly Journal of Economics, 120(2), 517-549.

Jones, C. I., AND D. SCRIMgeour (2008): “A New Proof of Uzawa's Steady-State Growth Theorem," Review of Economics and Statistics, 90(1), 180-182.

KLUmP, R., P. MCADAM, AND A. Willman (2007): “Factor Substitution and Factor Augmenting Technical Progress in the US: A Normalized Supply-Side System Approach," Review of Economics and Statistics, 89(1), 183-192.

SCHLICHT, E. (2006): “A Variant of Uzawa's Theorem,” Economics Bulletin, 5(6), 1-5.

UzAwA, H. (1961): "Neutral Inventions and the Stability of Growth Equilibrium," Review of Economic Studies, 28(2), 117-124. 